\title{
Emerging efforts for discovering new biomarkers of liver disease and hepatotoxicity
}

"To foster identification of more reliable biomarkers of liver disease and hepatotoxicity and their translation in clinical practice, this issue of Biomarkers in Medicine solicited expert research articles and reviews in development of biomarkers of liver disease and hepatotoxicity."

\section{KEYWORDS: biomarkers — discovery - hepatotoxicity = liver disease}

\section{Tremendous \& concerted effort has yet to provide the biomarkers needed for patient stratification}

The President's Council of Advisors on Science and Technology (PCAST) defines personalized medicine as "the tailoring of medical treatment to the individual characteristics of each patient. It does not literally mean the creation of drugs or medical devices that are unique to a patient, but rather the ability to classify individuals into subpopulations that differ in their susceptibility to a particular disease or their response to a specific treatment. Preventive or therapeutic interventions can then be concentrated on those who will benefit, sparing expense and side effects for those who will not" [101]. Discovering biomarkers with high sensitivity and specificity is a vital prerequisite for realizing personalized medicine in such clinical applications as diagnostics and disease classification, treatment selection and prognostics. Notable effort and success in developing requisite biomarkers has been seen within public, private and academic institutions alike, all aided by and providing more impetus for new molecular technologies [1].

The human liver is an organ critical to human survival and wellbeing, performing vital biological functions such as detoxification, protein synthesis and production of digestive enzymes. Exposure to drugs and environmental chemicals can cause liver damage and loss of liver function in part or even in whole. Liver diseases associated with hepatotoxicity are complex, and the molecular mechanisms are poorly understood and manifest in a genetically dependent manner. Diagnosis of liver diseases and detection of hepatotoxicity, especially in early stages, have long been established as paramount goals driving major, relentless and often concerted research efforts. Success in discovering effective biomarkers for liver disease and hepatotoxicity, and the ability to stratify populations in accordance with vulnerability, would be invaluable. Numerous prospective biomarkers of liver disease and hepatotoxicity have been explored, but only a handful have survived to be developed, qualified, approved and then deployed in clinical practice. Biomarkers in the clinic have narrow applicability as providers of cautionary signals, but lacking specificity, generally, and the ability to predict disease etiology, particularly [2]. Despite prodigious effort, 'gold-standard' biomarkers of liver disease and hepatotoxicity have not yet emerged [3]. The motivation of this issue of Biomarkers in Medicine is to bring together expert research articles and reviews in order to provide a contemporary overview of progress in discovery of biomarkers of liver disease and hepatotoxicity, as well as delineate the technology advances on which future advances depend.

Metabolites \& miRNAs have potential as noninvasive biomarkers of liver disease \& hepatotoxicity

Serum ALT, AST and ALP activities, and total bilirubin concentration, are examples of conventional biomarkers for liver disease and hepatotoxicity. Normally used in combination as generic indicators of liver injury, they fall short of the needed reliability and specificity. The search for alternatives has been a protracted effort by the pharmaceutical industry and scientific community. The search persists unabated as new promising approaches, metabolomics and miRNAs among them, supplant exhausted means of pursuit. This issue strives to shed light on more recent research approaches by providing reviews and research articles on them.

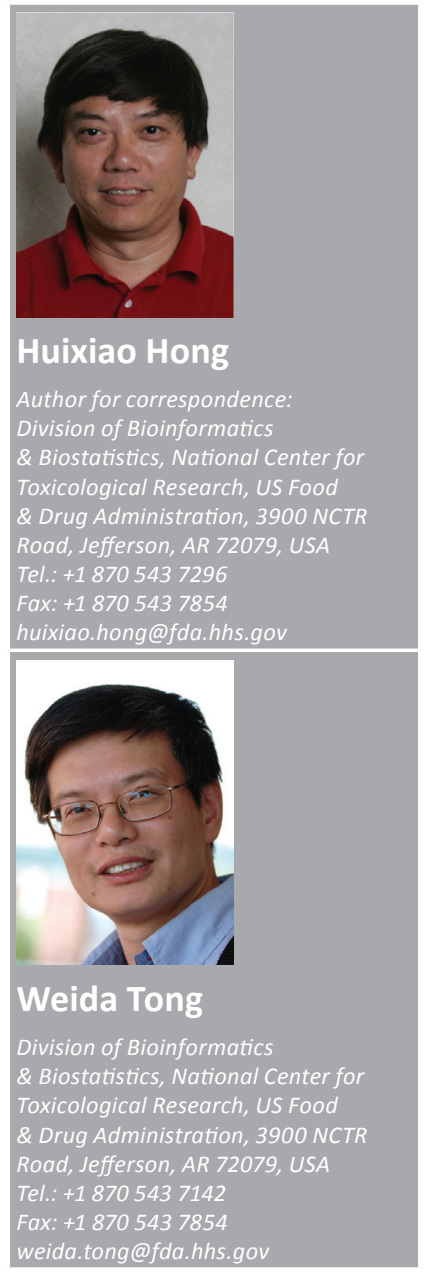

Future $\mathrm{fSS}$ 
Metabolomics studies nominally use mass spectroscopy and nuclear magnetic resonance spectroscopy to generate profiles of metabolites in a specific cellular process of an organ in order to identify metabolites with the potential to be biomarkers. The common analgesic acetaminophen (APAP), which is safe at therapeutic doses, is responsible for some $50 \%$ of acute liver failure cases owing to overdose [4]. Metabolomic profiling of APAP metabolism in mice revealed that acylcarnitines, intermediates in the mitochondrial $\beta$-oxidation of fatty acids, could provide a complementary biomarker for APAP-induced hepatotoxicity [5]. Bhattacharyya et al. recently examined the possibility of using serum acylcarnitines as biomarkers for APAP-induced hepatotoxicity in a clinical setting based on samples from 272 children by using ultra-performance liquid chromatography-tandem quadrupole mass spectroscopy [6]. APAP-induced hepatotoxicity was observed to be associated with long-chain acylcarnitines. Levels of oleoyl- and palmitoyl-carnitines were elevated in children with either therapeutic and/or overdose APAP exposure. Moreover, children with overdose APAP and with APAP protein adducts over the toxicity threshold had higher levels of acylcarnitines. While acylcarnitines in nonhospitalized children taking APAP need to be further investigated for evaluation of the clinical significance of acylcarnitine elevation following low-dose APAP exposure and, potentially, susceptibility to APAP-associated liver injury, the work demonstrates the potential for using metabolomics to discover biomarkers of liver disease and hepatotoxicity.

\section{"Translating miRNA signals into drug- induced liver injury biomarkers for the clinic requires more comprehensive studies on miRNA differential expression in patients treated with multiple classes of drugs known to have drug-induced liver injury potential..."}

miRNAs are sncRNA molecules composed of approximately 21 nucleotides. They play prominent roles in both pre- and post-translational gene regulation [7], and have been the subject of intensive research for use as both biomarkers [8] and therapeutic agent targets [9]. The recent availability of new technologies for profiling miRNAs has made the study of them as biomarkers of liver disease and hepatotoxicity possible [10]. Accordingly, we invited a panel of experts to review the requisite technologies and applications. Wang et al. reviewed the current technologies and research in using miRNAs as biomarkers of drug-induced liver injury (DILI), noting a number of challenges to be overcome [11]. Studies of circulating miRNAs leaked into serum after liver injury have been shown to have potential as biomarkers for DILI. Examples are miR-192 and miR-122, which performed better than the conventional DILI biomarkers such as ALT and AST [12]. miR-122 (but not serum ALT and AST) could effectively differentiate liver injury from extrahepatic injury. However, deriving effective biomarkers from circulating miRNAs will require further development to achieve practical clinical needs for simplicity, speed, robustness and sensitivity, along with reproducibility and acceptable specificity, all at low cost. Translating miRNA signals into DILI biomarkers for the clinic requires more comprehensive studies on miRNA differential expression in patients treated with multiple classes of drugs known to have DILI potential, followed by further technology advancement in miRNA detection to overcome aforementioned limitations. Fortunately, some ongoing projects address the needs, offering realistic expectations that more sensitive, specific, robust and cost-effective miRNA biomarkers of DILI will be forthcoming in the near future.

\section{In vitro assays based on human hepatocytes can accelerate discovery of biomarkers of hepatotoxicity}

Biomarkers of hepatotoxicity are usually identified and qualified using animal models. Qualified biomarkers are then explored for their possible translation into clinical practice by testing on humans. Almost every pharmaceutical company has programs developing such biomarkers, either unilaterally or in partnerships or other ventures [1]. Biomarker development is not only expensive because of animal use in the discovery phase, but because translation to clinical viability must address differences between animal models and humans. Striving to mitigate the substantial investment, researchers are increasingly using human cells in biomarker discovery and validation phases based on the premise that human cells retain in vivo properties. Using in vitro assays with human cells is markedly less expensive and more rapid. Moreover, the translation of biomarker discovered by using in vitro assays based on human cells may have less challenges as the difference between humans and human cells 
may not be larger than the difference between humans and animal models. Human hepatocytes have been widely used in identification of biomarkers of hepatotoxicity [13]. The goal of the review article by $\mathrm{Li}$ is to give an overview of the applications of in vitro assays based on human hepatocytes to the identification of biomarkers of hepatotoxicity [14].

\section{Leveraging the huge amount of publicly available data will facilitate translating biomarkers to the clinic}

DNA microarrays allow monitoring of the expression levels of tens of thousands of genes in a single experiment [15]. This technology has been widely used in development of biomarkers of liver disease and hepatotoxicity. Typically, an expression profile of tens of thousands genes is explored to extract signature genes that are associated with liver disease or drugs with DILI potential (similar mechanisms). Signature genes form biomarkers for diagnosis of liver disease, predicting DILI occurrence or investigating DILI mechanisms of new drugs. Many microarray data from liver disease and hepatotoxicity studies have been generated, and many putative biomarkers have been derived from those data. However, translation of those biomarkers to the clinic has been hampered by lack of thorough validation, as well as the limited accuracy and reproducibility observed in the biomarkers themselves. Encouragingly, novel methodologies for identification of genomic signatures of liver disease and hepatotoxicity have been developed that integrate the huge bolus of data that have been made publicly available. Hebels et al. reviewed a new method for discovering biomarkers of hepatotoxicity by deriving pathways from genomic databases [16]. Efforts to exploit the existing open access gene-expression data have relied on two types of databases: databases constructed using text-mining techniques such as the Comparative Toxicogenomics Database (CTD) [102]; and databases that collect gene-expression data such as ArrayExpress [103]. This review article introduced the databases and gave a systematic comparison of the two types of databases, particularly with regard to their utilization in biomarkers discovery. Performances of biomarkers derived from the two approaches were compared. Identifying biomarkers by means of deriving involved pathways seems an attractive alternative for translational research in development of biomarkers of liver disease and hepatotoxicity.

\section{Predictive models warrant applications of biomarkers in clinical practice \& drug development}

Biomarkers are often used to diagnose disease or to predict toxicity. Conventional biomarkers of liver disease and hepatotoxicity have a cutoff threshold for decision-making. By contrast, most recently discovered biomarkers are used together to make decisions in a more complicated way to render models for prediction or diagnosis. Biomarker validation comprises two different activities. First, the assay used to produce the data and the method to select biomarker feature (i.e., genes) must be qualified. Next, the biomarker must be qualified in the context of its intended use, such as disease diagnosis or toxicity prediction (e.g., of specific drugs). Because biomarker features are combined in an algorithmic model, the statistical analysis procedures used to construct composite predictive models should be qualified to evaluate applicability for the intended use. Many difficulties are encountered in discovering biomarker features to construct a robust predictor. One difficulty is multiplicity of potential features when using high-throughput/high-content analysis; that is, whether independent variable features are SNPs, genes, proteins or metabolites, the potential features will normally far outnumber the samples such that the risk of overfitting and selection of uninformative features (i.e., false positives) looms large. Although fusing different types of independent variables in a predictive model is becoming common, the risk of overfitting is even larger. Predictive models are vital to deployment of effective biomarkers. With the intent to emphasize the difficulties in developing and validating biomarkers of liver disease and hepatotoxicity, Chen et al. summarized most extant predictive models developed for predicting DILI in humans and systematically reviewed the current state of DILI predictive model development [17]. Pros and cons of different types of predictive models and methods were also discussed.

\section{New \& better biomarkers of liver disease $\&$ hepatotoxicity are anticipated}

Biomarkers are now being included in the labels of US FDA-approved drug products and are expected to play a pivotal role in advancing personalized medicine. With emerging efforts by researchers, clinicians, drug developers and regulators alike, it is anticipated that more and better biomarkers of liver disease and hepatotoxicity will be forthcoming. The path forward 
over the next few years will include the discovery of exploratory biomarkers, followed by efforts to incorporate them into drug development, regulatory decision-making and clinical practice. To foster identification of more reliable biomarkers of liver disease and hepatotoxicity and their translation in clinical practice, this issue of Biomarkers in Medicine solicited expert research articles and reviews in development of biomarkers of liver disease and hepatotoxicity. With the emerging efforts underway, we look forward to a brighter future for utilizing biomarkers of liver disease and hepatotoxicity in clinical practice and drug development and, ultimately, better medical care.

\section{Disclaimer}

The views presented in this article are those of the authors and do not necessarily reflect those of the US FDA. No official endorsement is intended or should be inferred.

\section{Financial \& competing interests disclosure}

The authors are employees of National Center for Toxicological Research, US FDA. The authors have no other relevant affliations or financial involvement with any organization or entity with a financial interest in or financial conflict with the subject matter or materials discussed in the manuscript apart from those disclosed.

No writing assistance was utilized in the production of this manuscript.

\section{References}

1 Hong H, Goodsaid F, Shi L, Tong W. Molecular biomarkers: a US FDA effort. Biomarkers Med. 4, 215-225 (2010).

2 Shi Q, Hong H, Senior J, Tong W. Biomarkers for drug-induced liver injury. Expert Rev. Gastroenterol. Hepatol. 4(2), 225-234 (2010).

3 Teschke R, Schwarzenboeck A, Hennermann $\mathrm{KH}$. Causality assessment inhepatotoxicity by drugs and dietary supplements. Br. J. Clin. Pharmacol. 66, 758-766 (2008).

4 Lee WM. Etiologies of acute liver failure. Semin. Liver Dis. 28, 142-152 (2008).

5 Chen C, Krausz KW, Shah YM et al. Serum metabolomics reveals irreversible inhibition of fatty acid beta-oxidation through the suppression of PPARalpha activation as a contributing mechanism of acetaminopheninduced hepatotoxicity. Chem. Res. Toxicol. 22(4), 699-707 (2009).

6 Bhattacharyya S, Yan K, Pence L et al. Targeted liquid chromatography-mass spectrometry analysis of serum acylcarnitines in acetaminophen toxicity in children. Biomarkers Med. 8(2), 147-159 (2014).
7 He L, Hannon GJ. MicroRNAs: small RNAs with a big role in gene regulation. Nature Rev. Genet. 5, 522-531 (2004).

8 Bartels CL, Tsongalis GJ. MicroRNAs: novel biomarkers for human cancer. Clin. Chem. 55, 623-631 (2009).

9 Nana-Sinkam SP, Croce CM. MicroRNAs as therapeutic targets in cancer. Trans. Res. 157, 216-225 (2011).

10 Liu J, Jennings $S$, Tong W, Hong $H$. Next-generation sequencing for profiling expression of miRNAs: technical progress and applications in drug development. J. Biomed. Sci. Eng. 4, 666-676 (2011).

11 Wang Y, Chen T, Tong W. miRNAs and their application in drug-induced liver injury. Biomarkers Med. 8(2), 161-172 (2014).

12 Laterza OF, Lim L, Garrett-Engele PW et al. Plasma microRNAs as diagnostically sensitive and specific biomarkers of tissue injury. Clin. Chem. 55(11), 1977-1983 (2009).

13 Persson M, Loye AF, Mow T, Hornberg JJ. A high content screening assay to predict human drug-induced liver injury during drug discovery. J. Pharmacol. Toxicol. Methods 68(3), 302-313 (2013).
14 Li AP. Biomarkers and human hepatocytes. Biomarkers Med. 8(2), 173-183 (2014).

15 Schena M, Shalon D, Davis RW, Brown PO. Quantitative monitoring of gene expression patterns with a complementary DNA microarray. Science 270, 467-470 (1995).

16 Hebels DGA, Jetten MJA, Aerts HJW et al. Evaluation of database-derived pathway development for enabling biomarker discovery for hepatotoxicity. Biomarkers Med. 8(2), 185-200 (2014).

17 Chen M, Bisgin H, Tong L et al. Toward predictive models for drug-induced liver injury in humans: are we there yet? Biomarkers Med. 8(2), 201-213 (2014).

\section{Websites}

101 President's Council of Advisors on Science Technology: Priorities for Personalized Medicine (2008). www.whitehouse.gov/files/documents/ostp/ PCAST/pcast_report_v2.pdf

102 Comparative Toxicogenomics Database. http://ctdbase.org

103 ArrayExpress. www.ebi.ac.uk/arrayexpress 